\title{
Optical characterization of CdTe/ZnTe semiconductor wires and dots
}

\author{
C. GOURGON, B. ERIKSSON, L.S. DANG, H. MARIETTE and C. VIEU*
}

CEA-CNRS joint group "Microstructures de Semiconducteurs II-VI", Laboratoire de Spectrométrie Physique, Université Joseph Fourier, BP. 87, 38402 Grenoble, France

* Laboratoire de Microélectronique et de Microstructures, CNRS, 196 av. H. Ravera, 92225 Bagneux, France

\begin{abstract}
Arrays of wires and dots have been fabricated by electron beam lithography and $\mathrm{Ar}^{+}$ion beam etching from $\mathrm{CdTe} / \mathrm{ZnTe}$ quantum wells. Low temperature photoluminescence coming out from these structures is still observed for the smallest wires $(40 \mathrm{~nm})$ whereas for the dots, the detection limit occurs for lateral dimensions of $100 \mathrm{~nm}$.
\end{abstract}

\section{INTRODUCTION}

With the development of wide gap II-VI semiconductor heterostructures for optoelectronic applications and short visible wavelengths [1][2], it is important to investigate the optical properties of II-VI quantum wires and dots structures in order to evaluate the predicted attractive properties of such nanostructures (increase of the exciton oscillator strength, lower laser threshold). For II-VI nanostructures only wires and dots made of $\mathrm{ZnSe}$ compounds have been reported so far in the literature [3][4]. In this paper we present the optical characterization of CdTe/ZnTe nanostructures fabricated by electron beam lithography and $\mathrm{Ar}^{+}$ion beam etching with lateral sizes down to $40 \mathrm{~nm}$ and $70 \mathrm{~nm}$ for wires and dots respectively. The total luminescence intensity in such nanostructures is comparable to the one obtained with the reference quantum well in the unetched part of the sample but is dominated by recombination of exciton localized on defects rather than by free exciton. Below $40 \mathrm{~nm}$ wire widths (100 nm dot widths) this extrinsic emission is completely quenched.

\section{FABRICATION}

The CdTe/ZnTe (001) quantum wells were grown by molecular beam epitaxy on GaAs substrate, covered by a $2.7 \mu \mathrm{m}$ thick
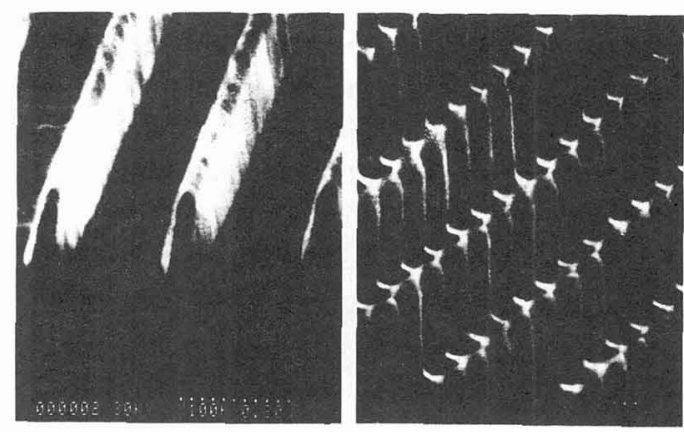

FIG.1. SEM images of periodic quantum wires and dots etched into the CdTe/ZnTe heterostructures. The five QWs are located below the $100 \mathrm{~nm} \mathrm{CdZnTe} \mathrm{cap} \mathrm{layer.} \mathrm{The} \mathrm{sizes} \mathrm{are} 40 \mathrm{~nm}$ for the wires and $70 \mathrm{~nm}$ for the dots. (The scale is given by two successive points which correspond to $30 \mathrm{~nm}$ ). 
CdZnTe (16\%Zn) buffer layer. The multiquantum wells (MQWs) structure contains 5 periods with QWs and barriers thicknesses of $13 \mathrm{~nm}$ and $1.6 \mathrm{~nm}$ respectively. A conventional $\mathrm{e}^{-}$beam nanolithography was used on a $150 \mathrm{~nm}$ thick polymethylmethacrylate resist layer to produce high density periodic patterns on the sample. The experimental conditions (exposure time, beam spot size) were optimized to obtain well defined arrays of $40 \mu \mathrm{m} \times 40 \mu \mathrm{m}$ of wires and dots. After deposition of a Titanium layer of $50 \mathrm{~nm}$, a lift-off process was used to produce metallic wires and dots. The transfer of this pattern to the underlying QWs was obtained by etching the sample through the Ti masks with a conventional $\mathrm{Ar}^{+}$ion beam etching system. The nanostructures were observed using a high resolution scanning electron microscope (SEM). Fig. 1 shows such images attesting that the technological processes (lithography and etching) allow us to control lateral dimensions down to $40 \mathrm{~nm}$ (respectively $70 \mathrm{~nm}$ ) for the wires (respectively dots). Moreover a closed examination of SEM images shows that the typical inclination of the sidewalls was about $10^{\circ}$. Such patterns were reproduced with various lateral sizes ( $40 \mathrm{~nm}$ to $170 \mathrm{~nm}$ for the wires and $70 \mathrm{~nm}$ to $210 \mathrm{~nm}$ for the dots) for a given period $(0.4 \mu \mathrm{m})$, and also as a function of the period $(0.4 \mu \mathrm{m}$ to $2 \mu \mathrm{m})$ for a given wire width $(80 \mathrm{~nm})$.

\section{OPTICAL CHARACTERIZATION}

Optical spectroscopy (photoluminescence, photoluminescence excitation) was performed at low temperature $(1.8 \mathrm{~K})$ with a mapping set-up having a spatial resolution of $20 \mu \mathrm{m}$ (laser spot size). The sample was excited either above the band gap of the barrier with an Ar laser or in resonance in the well using a dye laser with a typical power density of $500 \mathrm{~W} / \mathrm{cm}^{2}$. Fig. 2 shows PL spectra of quantum wires with lateral sizes changing from $170 \mathrm{~nm}$ to $40 \mathrm{~nm}$, together with a PL spectrum of quantum dots (210 $\mathrm{nm}$ thick) and a reference spectrum obtained on a large area 2D zone. This spectrum exhibits two lines: $\mathrm{X}$ line, corresponding to the $\mathrm{e}_{1} \mathrm{~h}_{1}$ intrinsic excitons in the CdTe-ZnTe QWs and $\mathrm{Y}$ line corresponding to extrinsic excitons localised on residual defects (its energy $-4 \mathrm{meV}$ below the $\mathrm{X}$ oneis compatible with a donor bound exciton [5][6]). Extrinsic luminescence at $1.57-1.58 \mathrm{eV}$ is probably related to extended defects [7]. The PL spectra of wide wires and dots as compared to the $2 \mathrm{D}$ one exhibit:

(i) a blue shift of both exciton lines towards higher energy $(\approx 1.5 \mathrm{meV}$ for the wires and $3 \mathrm{meV}$ for the dots),

(ii) a vanishing of the extrinsic luminescence at 1.57$1.58 \mathrm{eV}$,

(iii) a marked variation in the relative intensity of the intrinsic $e_{1} h_{1}$ and extrinsic transitions (ratio $X / Y$ ).

The above PL features are similar to the ones observed on the PL spectra of samples implanted at $300 \mathrm{~K}$ with different ions ( $\mathrm{Zn}, \mathrm{Ar}, \mathrm{Cd}$ ) following by a moderate annealing for which interdiffusion of CdTe/ZnTe strained QWs was evidenced [7]. In our case the ion beam etching with $\mathrm{Ar}^{+}$could create an annealing effect which would explain the blue shift

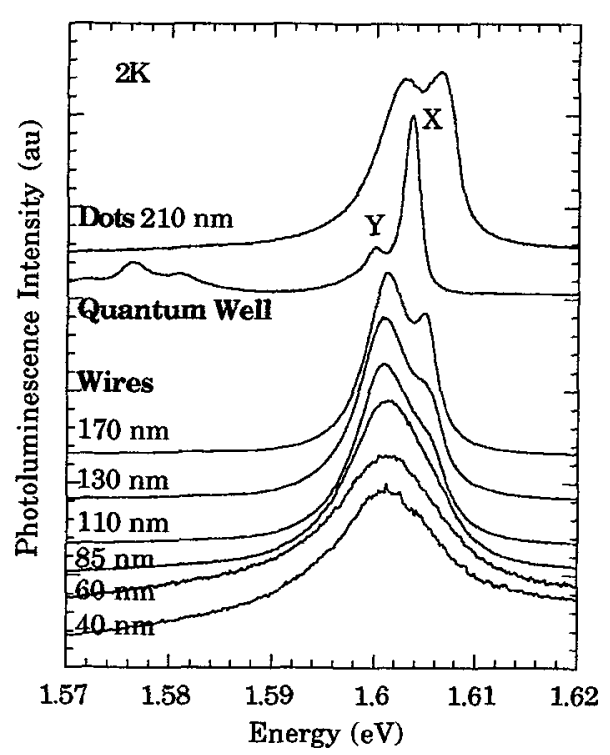

FIG.2. Photoluminescence spectra of set of wires with various sizes (170 nm to $40 \mathrm{~nm}$ ) together with a PL spectrum of quantum dots $(210 \mathrm{~nm})$ and of a reference 2D quantum well structure. The intensities of all the spectra are normalized to the maxima. 
observed for these exciton lines which can be due to some ion beam induced interdiffusion. This blue shift can not be due to strain relief effect because the fundamental CdTe gap $\left(e_{1} h_{1}\right)$ is not sensitive to biaxial (001) strain, as is the case here [8]. The vanishing of extrinsic luminescence at $1.57-1.58 \mathrm{eV}$ and the change in the ratio $\mathrm{X} / \mathrm{Y}$ were also observed in Ref. 7 as in the present case. The former effect, that is the increase of the $Y$ line intensity, can reveal a variation in the distribution of impurities or defects responsible for the extrinsic line $Y$ during the ion beam etching.

For narrower wires and dots, the intrinsic $\mathrm{X}$ line disappears and only the $\mathrm{Y}$ line can be detected. This line broadens up to $10 \mathrm{meV}$ for the smallest wire size $(40 \mathrm{~nm})$ and no significant blue shift due to lateral confinement is observed. In our case lateral confinement effect could be obscured by dispersion of wire lateral size in the MQW structure because of the inclined sidewalls. Taking wire sizes equal to $40 \pm 15 \mathrm{~nm}$, one would expect a blue shift varying between 2 and $7 \mathrm{meV}$ for an infinite lateral potential.

As far as the intensities are concerned, Fig. 3 shows the evolution of the $\mathrm{X}$ and $\mathrm{Y}$ exciton lines intensities for both wires and dots after deconvoluting the PL spectra. Let us mention that the PL results obtained for the $40 \mathrm{~nm}$ wires are not reported in this figure because they were obtained on an array with a different period and under a resonant excitation with a different power. The striking result is the increase of the $\mathrm{Y}$ line luminescence intensity for the wide nanostructures as compared to the one obtained for the reference 2D unetched part. The $\mathrm{X}$ and $\mathrm{Y}$ exciton lines intensities drop by about one order of magnitude for the smallest patterns. Note that no overgrowth was realized to obtain these results by contrast with the III-V nanostructures where the recovery of luminescence intensity was obtained by overgrowth of the etched patterns in order to reduce surface recombination [9]. Moreover no corrections of the PL intensities were made for the area filling factor: this is justified by comparing
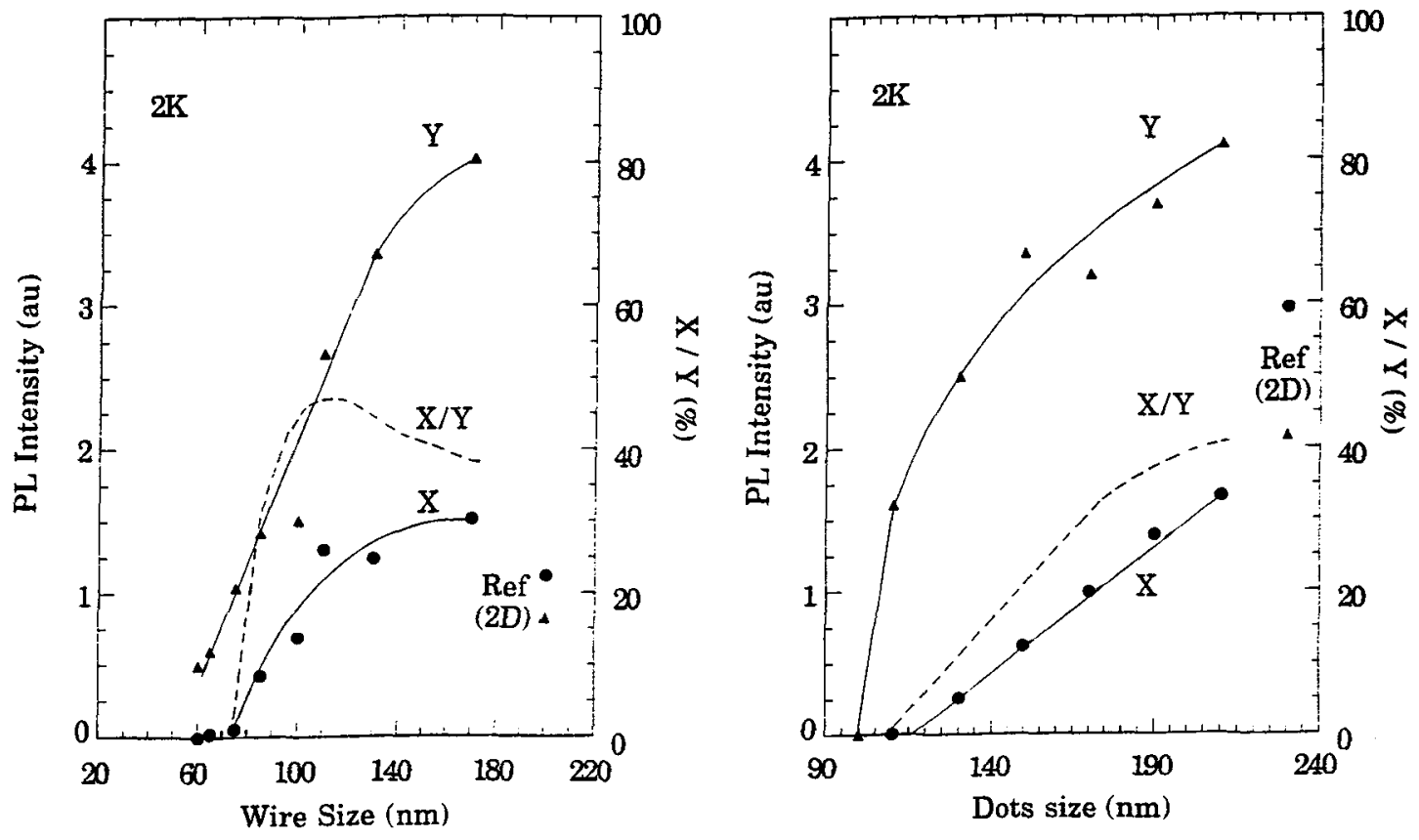

FIG.3. PL intensities of the two exciton lines as a function of the lateral dimensions. They are deduced from spectra obtained with a non resonant excitation. The circles correspond to the intrinsic recombination ( $\mathrm{X}$ line) and the triangles to extrinsic one ( $Y$ line). The full lines are only a guide for the eyes and the dashed lines are the experimental results of the intensity ratio $\mathrm{X} / \mathrm{Y}$. The $\mathrm{PL}$ intensities observed for the reference $2 \mathrm{D}$ unetched part are also shown. 
resonant excitation results which suggest that carriers created in the CdZnTe buffer layer between the wires (dots) diffuse into the wires (dots) and then recombine radiatively. The increase of the PL quantum efficiency in the wide nanostructures arrays is correlated with two experimental observations. First in the implantation enhanced interdiffusion study mentioned above [7] an increase by a factor 2 of the PL efficiency was observed between the $2 \mathrm{D}$ as-grown sample and the annealed one. Second PL intensity from arrays of $80 \mathrm{~nm}$ wires is increased by a factor of 4 when the period is smaller than 0.7 $\mu \mathrm{m}$. This grating effect could be due to the electrodynamic polarization effect as calculated in Ref. 10 .

Finally as shown on Fig. 3 the free exciton emission intensity ( $\mathrm{X}$ line) vanishes always before the extrinsic one ( $\mathrm{Y}$ line). This result can be due to the larger mobility of the free excitons which can migrate to the wire (dot) sidewalls and recombine non radiatively due to surface defects. On the other hand localized centers such as impurities into the wires (dots) can favour the observation of radiative recombination ( $\mathrm{Y}$ line) down to the smallest nanostructures sizes.

\section{CONCLUSION}

Quantum wires and dots are fabricated from $\mathrm{CdTe} / \mathrm{ZnTe}$ multiquantum wells grown by molecular beam epitaxy. High resolution electron beam lithography and $\mathrm{Ar}^{+}$ion beam etching are used to define arrays of wires and boxes with sizes down to $40 \mathrm{~nm}$ and $70 \mathrm{~nm}$ respectively.

The PL spectra and the PL intensities of these nanostructures are studied as a function of their size. Without any overgrowth the PL intrinsic exciton recombination (X line) is observed for wires (dots) as small as $80 \mathrm{~nm}(130 \mathrm{~nm})$. Moreover PL intensity from extrinsic excitons bound to localised centers ( $\mathrm{Y}$ line) can be detected for the smallest wires $(40 \mathrm{~nm})$ and for dots down to $100 \mathrm{~nm}$. These optical results, especially the PL efficiency which is not completely quenched by the surface recombination, are very promising to study optically the lateral confinement.

We are thankful to J.Cibert, K.Saminadayar and S.Tatarenko for providing the MBE growth sample.

\section{REFERENCES}

[1] Haase M.A., Qiu J., Depuydt J.M. and Cheng H., Appl.Phys.Lett. 59 (1991) 1272.

[2] Jeon H., Ding J., Patterson W., Nurmikko A.V., Xie W., Grillo D.C., Kobayashi M. and Gunshor R.L., Appl.Phys.Lett. 59 (1991) 3619.

[3] Walecki W., Patterson W.R., Nurmikko A.V., Luo H., Samarth N., Furdyna J.K., Kobayashi M., Durbin S.and Gunshor R. L., Appl.Phys.Lett. 57 (1990) 2641

[4] Foad M.A., Smart A.P., Wat M.T, Sotomayor Torres C.M., Khun W., Wagner P.H., Leiderer H., Bauer S., Wilkinson C.D.W., Gebhardt W. and Razeghi M., Proceedings.of the Int.Symp. on Nanostructures and Mesoscopic Systems, Santa Fe 19-24 May 1991.

[5] Mariette H., Dal 'bo F., Magnea N., Lentz G. and Tuffigo H., Phys.Rev. B 38 (1988)12443.

[6] Cox R.T., Mandray A., Huant S., Bassani F., Saminadayar K. and Tatarenko S., Mat.Sci.Engi. B 16 (1993) 83.

[7] Hamoudi A., Cibert J., Feuillet G., Jouneau P.H., Le Si Dang, Ligeon E., Pautrat J.L., Saminadayar K. and Tatarenko S., J.Appl.Phys. 74 (Aug. 1993).

[8] Merle d'Aubigné Y., Mariette H., Magnea N., Tuffigo H., Cox R.T., Lentz G., Le Si Dang, Pautrat J.L. and Wasiela A., J. Crystal Growth 101 (1990) 650.

[9] See for example: -lzrael A., Sermage B., Marzin J.Y., Ougazzaden A., Azoulay R., Etrillard J., ThierryMieg V. and Henry L., Appl.Phys.Lett. 56 (1990) 830., -Wang P.D., Sotomayor-Torres C.M., Benisty H., Weisbush C. and Beaumont S.P., Appl.Phys.Lett. 61 (1992) 946., -Pieger K., Gréus C.H., Straka J. and Forchel A., 7th EuroMBE, Bardonecchia 7-10 March 1993.

[10] Bockelmann U., Europhysics Lett. 16 (1991) 601. 